\title{
Directed emission of CdSe nanoplatelets originating from strongly anisotropic 2D electronic structure
}

Riccardo Scott, ${ }^{\dagger}, \S$ Jan Heckmann ${ }^{\dagger}, \S$ Anatol V. Prudnikau, ${ }^{\ddagger}$ Artsiom Antanovich, ${ }^{\ddagger}$ Aleksandr Mikhailov, ${ }^{\ddagger}$ Nina Owschimikow, ${ }^{\dagger}$ Mikhail Artemyev ${ }^{\ddagger}$ Juan I. Climente, Ulrike Woggon, ${ }^{\dagger}$ Nicolai B. Grosse, ${ }^{\dagger}$ and Alexander W. Achtstein ${ }^{* \dagger}$

$\dagger$ Institute of Optics and Atomic Physics, Technical University of Berlin, Strasse des 17. Juni 135, 10623 Berlin, Germany

$\ddagger$ Research Institute for Physical Chemical Problems of Belarusian State University, 220006, Minsk, Belarus

IDepartament de Química Física i Analítica, Universitat Jaume I, E-12080, Castelló de la Plana, Spain

$\S R$. Scott and J. Heckmann contributed equally to this work

E-mail: achtstein@tu-berlin.de

Fax: $+49(0) 3031421079$

Intrinsically directional light emitters are potentially important for applications in photonics including lasing and energy-efficient display technology. Here we propose a new route to overcome intrinsic efficiency limitations in light emitting devices by studying a CdSe nanoplatelets monolayer that exhibits strongly anisotropic, directed photoluminescence. Analysis of the two-dimensional kspace distribution reveals the underlying internal transition dipole distribution. 
The observed directed emission is related to the anisotropy of the electronic Bloch states governing the exciton transition dipole moment and forming a bright plane. The strongly directed emission perpendicular to the platelet is further enhanced by the optical local density of states and local fields. In contrast to the emission directionality, the off-resonant absorption into the energetically higher 2D-continuum of states is isotropic. These contrasting optical properties make the oriented CdSe nanoplatelets, or superstructures of parallel-oriented platelets, an interesting, and potentially useful class of semiconductor-based emitters.

Light-matter interaction and emission characteristics in semiconductors are mediated by the transition dipole moment (TDM). It is determined by the Bloch functions (states) associated with the bands involved in an optical transition and the envelope function defining the selections rules. ${ }^{1}$ In theory, the properties of optical TDMs in $2 \mathrm{D}$ materials such as II-VI nanoplatelets, ${ }^{2,3}$ nanobelts ${ }^{4}$ and 2D transition metal dichalcogenides ${ }^{5}$ can be highly anisotropic $^{3,6-16}$ analogous to those in molecules. Until now, however, very few reports support this hypothesis apart from one study on $2 \mathrm{D} \operatorname{MoS}_{2} \cdot{ }^{16}$ We present a combined experimental and theoretical approach to obtain quantitative information about the Bloch functions of 2D materials. Using CdSe nanoplatelets we demonstrate that, in contrast to a resonantly excited molecular system, ${ }^{17}$ the absorption is due to an isotropic transition dipole distribution whereas the heavy hole exciton in CdSe platelets has a highly anisotropic transition dipole distribution forming a bright plane that coincides with the platelet plane. Using 2D $k$-space spectroscopy we show the correlation between the directional external radiation pattern of an oriented sample of 2D CdSe nanoplatelets and the anisotropic intrinsic TDM distribution, and thus the underlying Bloch functions. Our theoretical analysis shows that the semiconductor Bloch functions are strongly anisotropic and hence directional for heavy hole excitons, and isotropic in the continuum where heavy hole $(h h)$, light hole $(l h)$ and the split-off (so) bands contribute. This sets the platelets apart from systems, in which an intrinsically isotropic emission is re-shaped only by the dielectric contrast and the external 
photon density of states. ${ }^{18}$

Beyond intrinsic electronic effects, the anisotropic shape of the nanoplatelets induces dielectric effects, further concentrating the intrinsically enhanced emission perpendicular to the platelet. This is a fundamental advantage over (elongated) wurtzite CdSe quantum dots where the bright plane does not coincide with the dielectrically favoured axis. ${ }^{19-22}$ Therefore, CdSe nanoplatelets can serve as efficient directional emitters for photonic applications such as display technology.

\section{$2 \mathrm{D} k$-space spectroscopy}

a

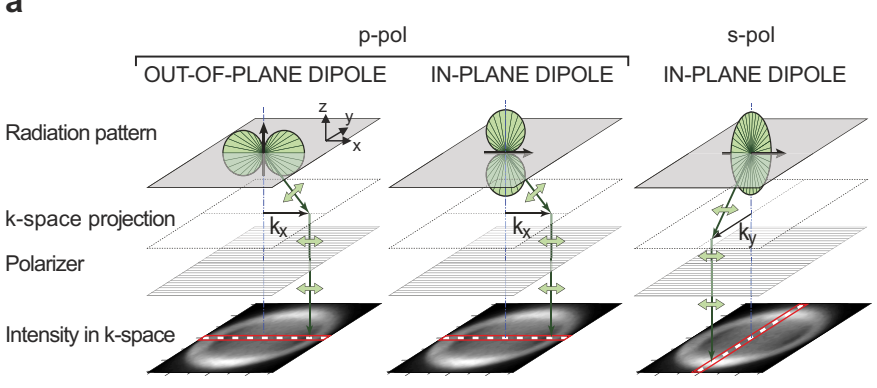

b

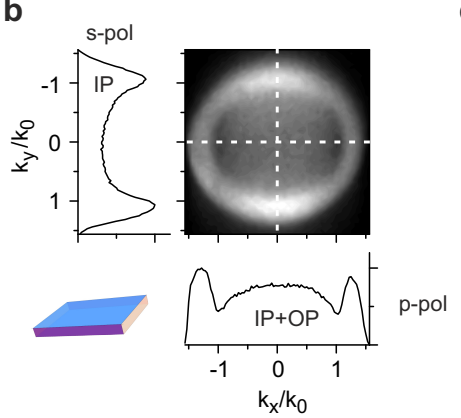

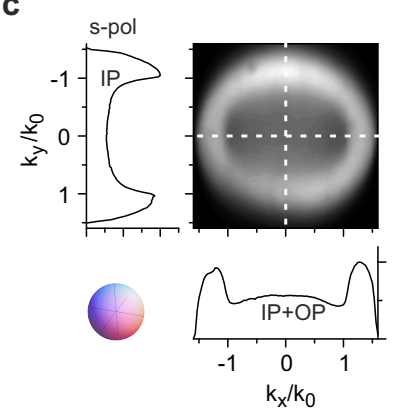

Figure 1: Momentum ( $\boldsymbol{k}$-space) resolved photo-luminescence: (a) By choosing one polarisation axis, the back aperture image of a microscope objective can be analyzed with respect to in-plane (IP) and out-of-plane (OP) transition dipole emission. We define radiation where $\vec{E}$ and $\vec{k}$ coincide in the $x-z$-plane as p-polarized. Radiation with $\vec{E}$ pointing in $x$ direction and $\vec{k}$ lying in the $y-z$-plane is thus s-polarized. ${ }^{23,24}$ Cuts in $k_{x}$ or $k_{y}$ direction are well defined and reduce the problem to a 2D geometry. (b) $k$-vector dependent emission of a monolayer of oriented nanoplatelets and (c) a monolayer of dots. The profiles attached to the images display cuts in $k_{x}(\mathrm{p}-\mathrm{pol})$ and $k_{y}$ (s-pol). The latter contain only emission from IP oriented dipoles. Cuts in $k_{x}$ reveal the relative occurrence of IP and OP dipoles, i.e. the anisotropy of the dipole distribution. 
Figure 1 a shows the concept of our experiment performed at room temperature. An in-plane $(x-y)$ oriented monolayer of zincblende (ZB) CdSe nanoplatelets with random azimuthal orientation was deposited on fused silica substrates by a Langmuir technique (see Methods). Our emitters placed in the sample plane ( $x-y$ plane) may have out-of-plane (OP) z-oriented dipoles and in-plane (IP) dipoles. The sample is introduced in an inverted microscope-like setup yielding a three layer system consisting of substrate/(ligands and nanocrystals)/air. Our setup allows an angle- and subsequently $k$-vector dependent excitation and detection of the photoluminescence (PL) signal. $k_{x}$ and $k_{y}$ projections contain information about orientation of the dipoles (see Fig. 1 a). A monolayer of randomly oriented CdSe dots (ZB structure) with a similar emission energy to the platelets' serves as an isotropically absorbing and emitting reference ensemble.

Figure $1 \mathrm{~b}$ and $\mathrm{c}$ show the results of the $k$-vector resolved emission of oriented CdSe nanoplatelets and a CdSe quantum dot reference (both excited at normal incidence). The measured intensity profiles of the $k_{x^{-}}$and $k_{y^{-}}$-projections are plotted next to the CCD images, where dashed lines indicate the selected regions (cuts). The $k$-scale is normalized to the wavevector in air $\left(k_{0}=\omega / c\right)$. Radiation with a wave vector $\left|k_{x, y}\right|>k_{0}$ cannot couple into air resulting in increased reflected intensity collected by the objective for these $k$-vectors.

The $k$-dependent emission profile is considerably different for platelets and dots and can be related to a different fraction of IP and OP transition dipoles. The s-polarization projections $\left(k_{y}\right.$-cut) originate only from IP dipoles: their intensity profile merely depends on the dielectric function and thickness of the nanocrystal monolayer (see Supporting Information (SI) section D). We concentrate on cuts in $k_{x}$ direction as only p-polarization $I\left(k_{x}^{\text {out }}\right)$ contains signal from in- as well as out-of-plane dipoles. Also for addressing IP and OP dipoles in our 2D $k$-space spectroscopy the excitation beam is p-polarized and we scan the excitation $k$-vectors along $k_{x}^{i n}$.

In order to disentangle the contributions of the intrinsic dipole orientation (IP to OP ratio) and the external dielectric effects, we model the $k$-vector dependent platelet emission 

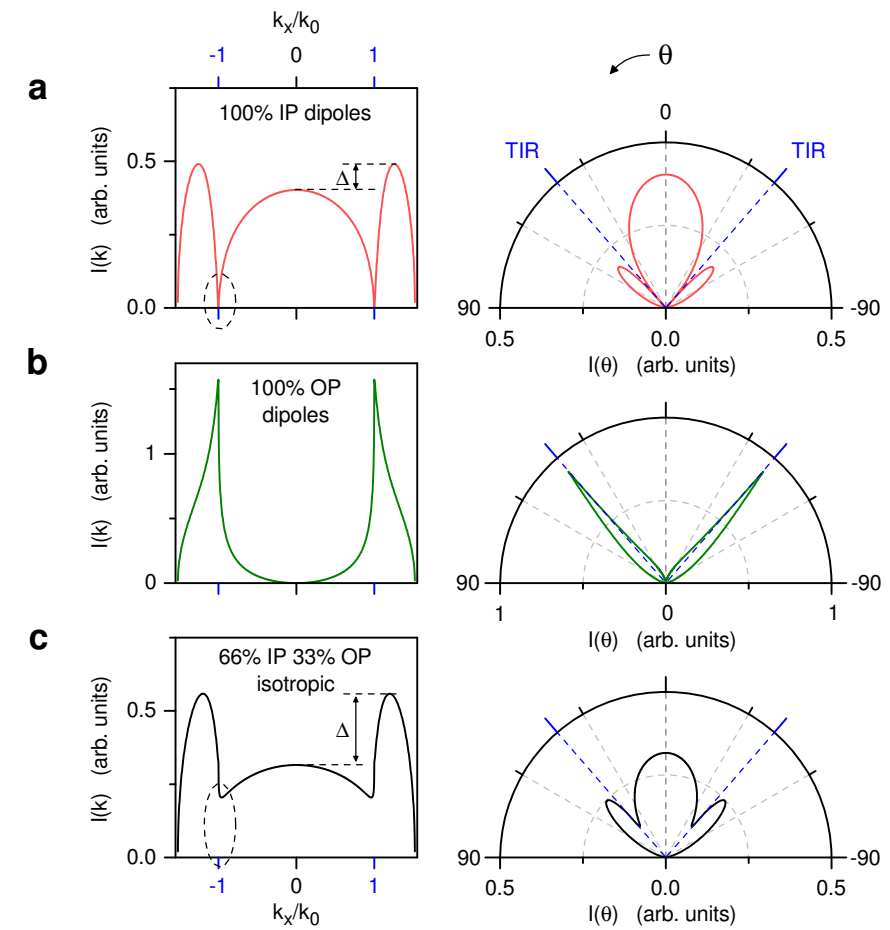

Figure 2: Calculated $k$-space spectra of pure in-plane (a) and out-of-plane (b) transition dipoles and an isotropic distribution of them (c). Left column: p-polarized spectra in $k$-representation. Right column: corresponding polar plots as a function of the angle $\theta$ with respect to the surface normal of the sample plane. Angles and wave vectors are related by $k_{x}^{a}=\sin \left(\theta_{a}\right) \cdot \sqrt{\epsilon_{1}^{a}} \omega^{a} / c$ with $a=i n$, out and the permeability $\epsilon_{1}^{a}$ of the glass substrate at the emission (out) or excitation (in) frequency $\omega^{a}$. The Jacobian conversion satisfying $\int I(k) \mathrm{d} k=\int I(\theta) \mathrm{d} \theta$ is taken into account. ${ }^{25}$ The case for emission is shown. Excitation is very similar as dispersion effects do not strongly alter the spectra. Compared to (a) the OP dipole contribution in the isotropic case (c) leads to an increase of the height difference $\Delta$ of the outer and inner maxima and a less pronounced minimum at $\left|k_{x} / k_{0}\right|=1$ (the angle of total internal reflection).

and excitation by using a local density of states formalism, ${ }^{16,24}$ extending it to anisotropic effective media using a Lorentz local field and effective medium approach. (see Methods)

Figure 2 shows the theoretical predictions for pure IP transition dipoles (row (a)), pure OP transition dipoles (b) at the emission energy. For an isotropic TDM distribution, shown in (c), $67 \%$ IP to $33 \%$ OP is expected, due to two degenerate IP dipole orientations in $x$ and $y$-direction and one OP dipole orientation in $z$-direction. The formalism for emission and excitation spectra is analogous due to Lorentz reciprocity. ${ }^{17}$ 44For a hypothetical identical TDM distribution for continuum excitation and lowest exciton emission, the forms of the k- 
space spectra would differ only slighty due to dispersion in the permittivities of the materials involved. Therefore the following discussion is valid for the PL emission intensity as a function of the excitation wave vector $k_{x}^{\text {in }}$ as well as for the emission spectra $I\left(k_{x}^{o u t}\right)$.

The left column of Figure 2 displays the $k$-space dependent luminescence intensity $I(k)$ while the right the corresponding polar representation.

The two side lobes in the pure IP dipole emission pattern correspond to modes beyond the angle of total internal reflection (TIR) of the glass to air interface $\left(\left|k_{x}\right| / k_{0}=1\right)$. They can be observed with our objective due to the index matching immersion optics used. A perfectly in-plane oriented transition dipole has no electric field component in the z direction and we thus expect no emission or absorption around the TIR angle, as seen in (a). In contrast, pure OP dipoles can only interact with the light field in a small region of $k$-vectors (angles) seen as two lobes around $\left|k_{x} / k_{0}\right|=1$ (TIR angle), see (b).

An important characteristic of $k$-space spectra is the height difference $\Delta$ shown in Figure $2 \mathrm{a}$ and $\mathrm{c}$ between the side lobes and the local maximum at $\left|k_{x}\right| / k_{0}=0$. It increases with increasing OP contributions. Furthermore, the contribution of OP dipoles in an isotropic distribution of transition dipoles (Figure 2c) leads to shallower dips at $\left|k_{x}\right| / k_{0}=1$ as compared to the pure IP case.

Figure $3 \mathrm{a}$ and $\mathrm{b}$ show false color plots of the measured p-polarized emission $I\left(k_{x}^{o u t}\right)$ as a function of the excitation wave vector $\left(k_{x}^{i n}\right)$ for platelets (a) and for the CdSe QD reference sample (b). These 2D $k$-space maps are obtained by plotting the cut in $x$ direction of the back aperture image (see Figure $1 \mathrm{~b}$ and c) for every excitation angle $k_{x}^{i n}$. The QDs' 2D $k$-space maps are symmetric, the NPLs' less so, which is a first indication that NPLs have different transition dipole distributions for absorption and emission (absorption and (PL) emission are measured at different energies, see Figure 4).

From the horizontal (excitation) and vertical (emission) cross sections we find that the angular emission changes in overall intensity, but its shape is independent of the excitation angle $\left(k_{x}^{i n}\right)$. According to the discussion above this indicates that different dipoles are in- 

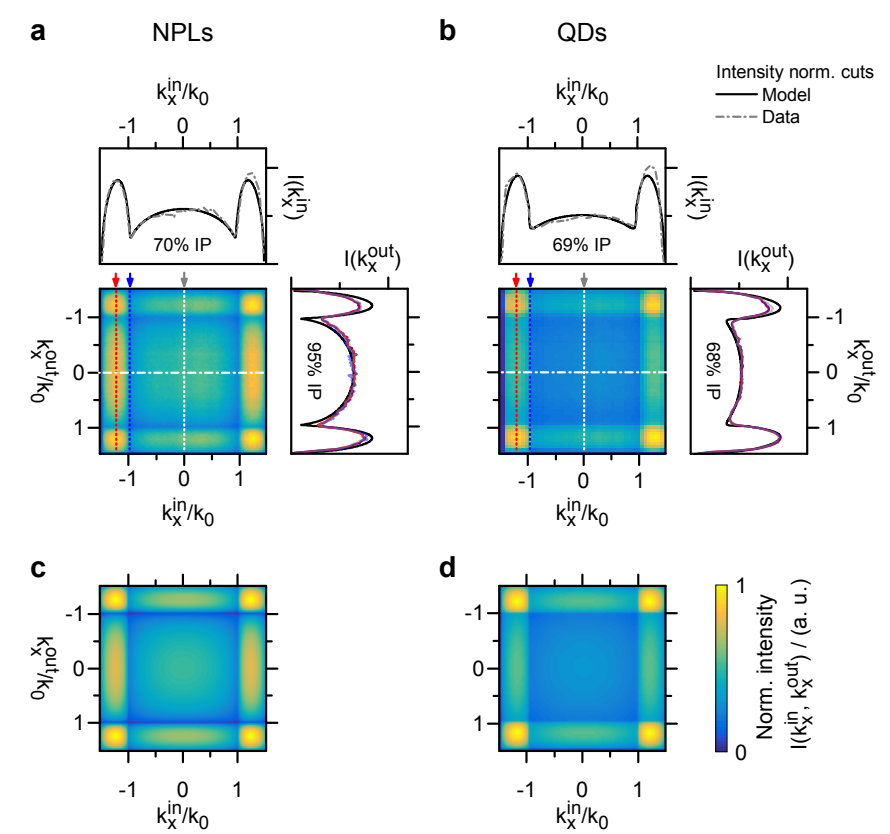

Figure 3: 2D $k$-space spectra of CdSe nanoplatelets and quantum dots. (a) and (b) False-color plots obtained by plotting the measured p-polarized emission $I\left(k_{x}^{\text {out }}\right)$ for every excitation wave vector $\left(k_{x}^{i n}\right)$. Cross-sectional plots on top of 2D plots: Intensity normalized horizontal cross section, i.e. emission at $k_{x}^{\text {out }} / k_{0}=0$ as a function of excitation. Plots to the right: Intensity normalized vertical cross sections at: $k_{x}^{i n} / k_{0}=-1.23,-1$ and 0 , red, blue gray, respectively. Fit curves are added as black lines as well as the resulting in-plane (IP) transition dipole contributions. We find highly anisotropic distribution of emitting NPL dipoles. (c) and (d): Calculated 2D $k$-space spectra for platelets and dots.

volved in the absorption process at $415 \mathrm{~nm}$ and the emission at $513 \mathrm{~nm}$. Thus the electronic system has no 'memory' of the excitation field's polarization.

As seen in Figure 3 a for nanoplatelets the height difference $\Delta$ is smaller for emission compared to excitation. Furthermore, the dip around the TIR angle $\left|k_{x}\right| / k_{0}=1$ for platelet emission $I\left(k_{x}^{\text {out }}\right)$ is more pronounced than for excitation. Following the discussion in Figure 2, nanoplatelets must have a lower fraction of emitting OP transition dipoles.

To quantify this conclusion, we show fitting results as black solid curves in Figure 3 a and b and as 2D $k$-space maps $I\left(k_{x}^{i n}, k_{x}^{o u t}\right)$ in c and d for NPLs and dots, respectively. All model parameters are determined by literature values and experimental conditions and are listed in Table I of the SI. The only free parameters are the dipole distributions, i.e. the ratio of IP to OP dipole moments in absorption and emission (section D of the SI). 
For both NPLs and dots we observe an excellent agreement of the model with the experimental results for emission and excitation (Fig. 3). The fit of the absorption characteristics of nanoplatelets (dots) yields 70\% (69\%) IP and 30\% (31\%) OP contributions. Also for the emission of CdSe dots we observe a nearly ideal isotropic $68 \%$ IP to $32 \%$ OP ratio.

However, the angular dependent emission of CdSe nanoplatelets is considerably distinct from their absorption. From the least-square fit we deduce a 95\% IP to 5\% OP distribution of the transition dipoles for the CdSe nanoplatelets' heavy hole emission. All values given above have an absolute error margin determined as $\pm 5 \%$ (see SI section D).

Due to the dominant contribution of IP transition dipoles the emission of CdSe platelets is concentrated strongly along the surface normal, as is visualized in comparison with the isotropic pattern radiated by QDs in panels a and c of Fig. 4 for p-polarized excitation (blue frame) and emission (green frame). Figure $4 \mathrm{~b}$ shows that the CdSe platelets are excited in the 2D absorption continuum, as the expected step like, constant density of states is observed at the excitation laser energy. For CdSe dots it is also known that an excitation energy of $2.99 \mathrm{eV}(415 \mathrm{~nm})$ leads to continuum state absorption where the excited carrier states do not depend on the spatial quantum confinement, Figure $4 \mathrm{~d}$.

For the platelets, the ratio of the intensity within the angle of total internal reflection compared to the intensity of the side lobes increases from 3.5 (excitation) to 5.0 (emission) in Figure 4 (a). Therefore CdSe NPLs can be considered as highly directional emitters with enhanced out-coupling efficiency. The strong anisotropic confinement has induced a predominant orientation of the TDM in a bright plane that coincides with the nanoplatelets' plane. The insets in Figure $4 \mathrm{~b}$ and d show the actual TDM distributions for all cases. The measured $95 \%$ IP contribution for CdSe platelets leads to a strongly oblate shape, reflecting the bright plane. 
a
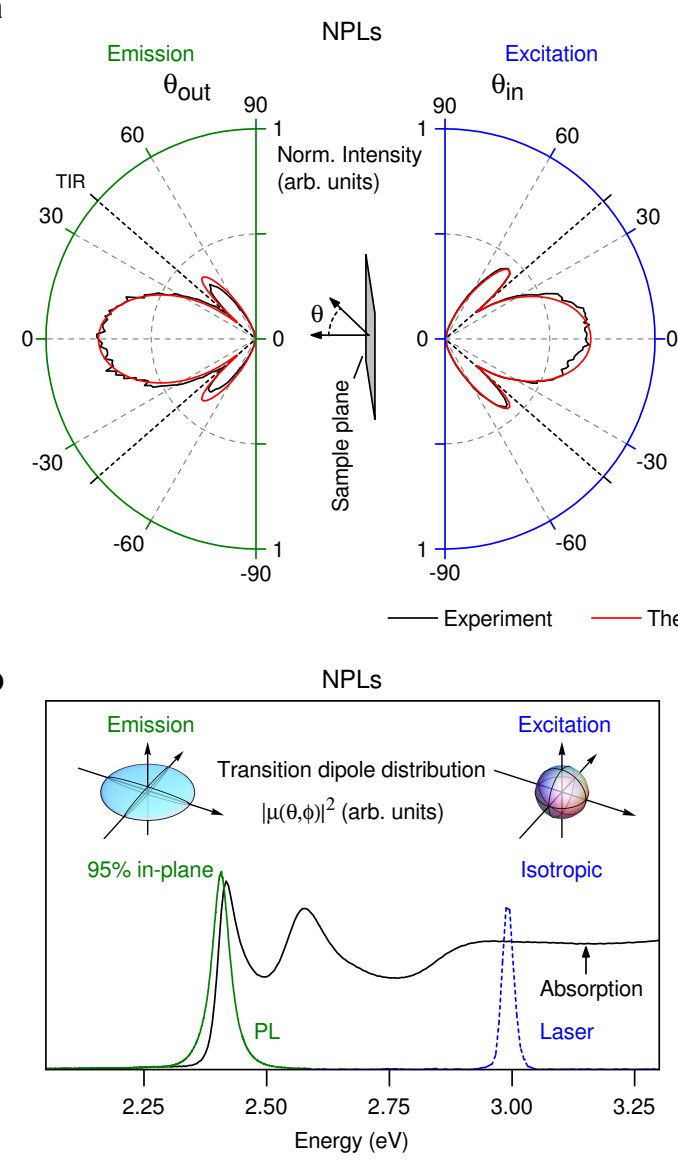

c

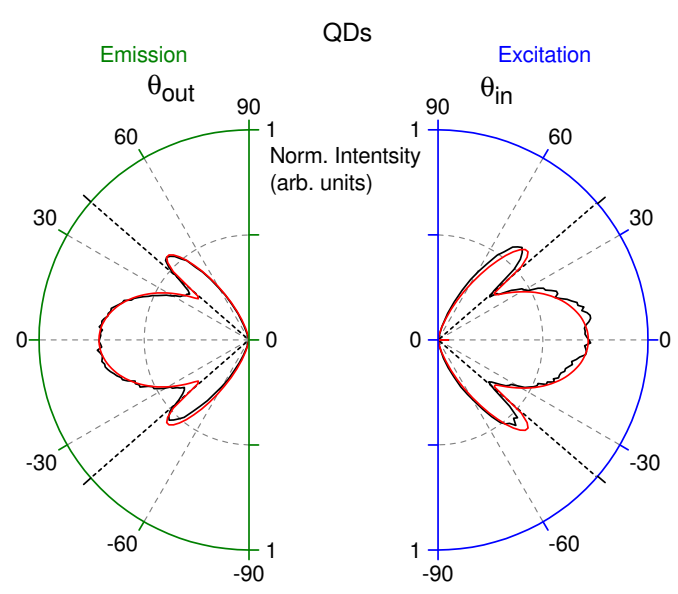

Angle of total internal reflection

d

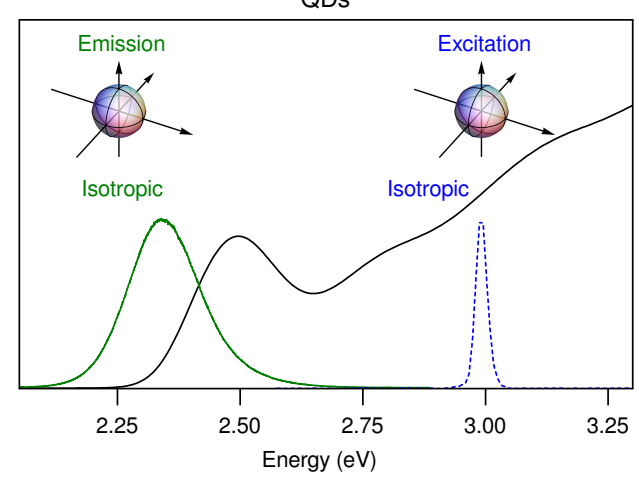

Figure 4: Angular dependent p-polarized emission and excitation of CdSe nanoplatelets and spherical quantum dot monolayers. (a) and (c): Polar plots of the angular dependent emission ( $\theta_{\text {out }}$, green frame) and the excitation $\left(\theta_{\text {in }}\right.$, blue frame) along with theory curves. Data and model are normalized to their total intensity, i.e. area under curve. The emission is measured for $\theta_{i n}=0$. The excitation curves are obtained by integrating the detected emission over $\theta_{\text {out }}$ ( (b) and (d): Absorption and photoluminescence spectra of the studied nanoplatelets and QDs along with the exciting laser. Insets: distributions of dipole moments for random orientation and for the strong anisotropic case of the emission of the oriented NPL monolayer calculated from our model.

\section{Radiation pattern: Bright plane and dielectric effects}

Directionality of the radiated field is desired in many photonics applications. Cubic bulk semiconductors used in modern photonics radiate isotropically. A directionality is then often imprinted by external dielectric effects or a manipulation of the external density of states for the light field. Our platelets, however, are intrinsically directional based on their electronic system. 
For example, light-extraction efficiency in quantum-well LED and organic LED (OLED) technology suffers from high contributions of z-polarized radiation (transverse magnetic $(\mathrm{TM})) .{ }^{26-29}$ Compared to transverse electric (TE) polarized light, it is more difficult to extract TM polarized light as it propagates predominantly in the lateral direction and undergoes total internal reflection, limiting the extraction efficiency ${ }^{29}$ in AlGaN-based LEDs to below $10 \% .{ }^{26}$ In our system OP transition dipoles, are related to TM (z-) polarized light, whereas TE polarized light stems from IP transition dipoles (Figure 2). Our NPLs have (nearly) only IP TDMs in emission. Thus the out-coupling of radiation (within the TIR angle) is strongly enhanced compared to an isotropic emitter (see Figure $4 \mathrm{a}$ and c). Hence NPLs and comparable 2D materials are promising candidates for efficient LED technology, boosting the out-coupling efficiency. In particular, ZB CdS ${ }^{30}$ nanoplatelet based LEDs with adjustable UV emission may overcome the fundamental light-extraction efficiency limitations ${ }^{26}$ of AlGaN-based LEDs.

The radiation intensity patterns derived from convolving the TDM distribution with the dipole radiation pattern (Figure $5 \mathrm{a}$ ) of an oriented platelet monolayer radiating in an isotropic medium is shown in Figure 5 b. It demonstrates the case without dielectric contrast, resembling excitons radiating into a virtually infinite CdSe medium. This pure electronic contribution to the radiation pattern is shown in blue for NPLs and in orange for dots. In contrast, the radiation emission pattern of a single transition dipole (like in many organic molecules ${ }^{17}$ ) is not directional, see Hertzian radiation pattern in Fig. 5 a. Clearly, the emission resulting from two degenerate dipoles forming a bright plane is far more directional, see Fig. 5 b.

Now we consider an isotropic surrounding, typically oleic acid ligands $(n=1.46),{ }^{31}$ with dielectric contrast to the CdSe nanocrystals. The dielectric effects are described by local field factors $f_{x, y, z}$ of the NPLs ${ }^{32}$ at the exciton emission energy. By averaging over the two in-plane components on the long axes of the platelets $f_{x}$ and $f_{y}$ we take the azimuthal random orientation of the NPL monolayer ensemble into account and construct the field 
a

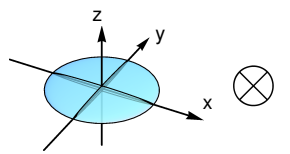

$|\mu(\theta, \phi)|^{2}$ (arb. units)

Transition dipole distribution

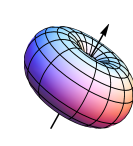

Dipole radiation pattern b

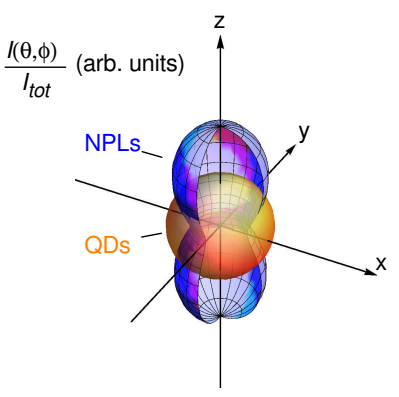

Intrinsic directionality (in CdSe)
C

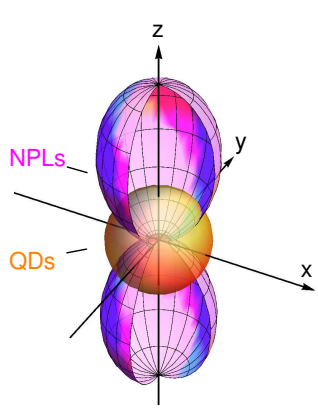

Enhanced directionality (in oleic acid) d

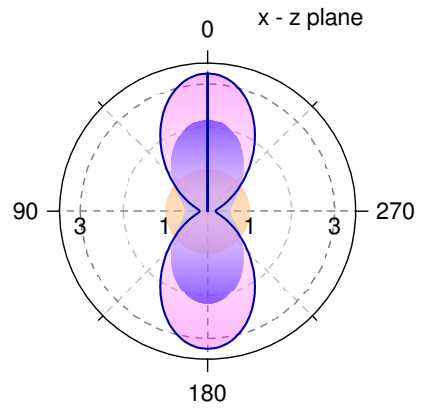

Figure 5: Radiation patterns of nanoplatelet and quantum dot monolayer ensembles in isotropic media. (a) The transition dipole distribution $|\mu|^{2}(\theta, \phi)$ (shown here for platelets as derived from the fits in Figure 4) is convoluted with the radiation pattern of a Hertzian dipole. The nanoparticle monolayers are oriented in the $\mathrm{x}, \mathrm{y}$ plane. (b) The obtained radiation intensity pattern of nanoplatelets (blue) and dots (orange) of the pure electronic contribution, as if the emitters were embedded in an infinite CdSe medium. (c) The external radiation patterns of platelets (magenta) and dots (orange) are calculated for an isotropic dielectric surrounding of oleic acid (with $n=1.46$ ) taking local field factors into account. The data in (b) and (c) is normalized to the total intensity $I_{\text {tot }}$ emitted over $4 \pi$. (d) Comparison of radiation patterns of dots (orange) and NPLs with and without dielectric effects (blue and magenta) in the $x, z$ plane. Clearly, platelets emit predominantly in the direction of the surface normal, which corresponds to 0 and 180 degrees. Dielectric effects (magenta) further enhance this directionality.

factor ellipsoid $|f|^{2}(\theta, \phi)$ (see SI section E). The product ${ }^{18,33}$ of the field factor ellipsoid and the dipole distribution $|\mu(\theta, \phi)|^{2}$ is convoluted with the radiation pattern of a Hertzian dipole. The obtained angular dependent radiation intensity of an emitter in an isotropic dielectric medium is shown in (c) for NPLs (magenta) and QDs (orange). Our platelets have a highly anisotropic shape and high dielectric contrast to their typical ligand or polymeric surrounding. Their in-plane local field factors $\left(\left|f_{I P}\right|^{2}\right)$ exceed those of the out-of-plane direction $\left(\left|f_{O P}\right|^{2}\right)$ by a factor $\sim 9$. The radiation from in-plane dipoles, i.e. the bright plane, is thus favored by the dielectric effects. The anisotropic local density of $\operatorname{states}^{34}$ related to the anisotropic local fields leads to a further concentration of the IP dipole emission perpendicular to the platelet plane. Thus in a realistic environment the emission becomes even more directed as seen in Figure 5(c). On the other hand, CdSe QD monolayer ensembles 
emit isotropically since they have both an isotropic dipole orientation and local field factors.

The cut through the $\mathrm{x}-\mathrm{z}$ plane of the radiation patterns is plotted in Figure $5(\mathrm{~d})$. In a surrounding with no dielectric contrast to CdSe (blue curve) the intensity per unit solid angle emitted perpendicular to the platelet is $\sim 4$ times higher than in-plane $(\mathrm{x}-\mathrm{y})$. When considering dielectric effects in a typical surrounding (magenta) this value reaches $\sim 16$. We conclude from Figure 5 that the emission of the CdSe nanoplatelets is highly directed normal to the platelet plane due to the existence of a bright plane of IP dipoles. This electronic effect is further intensified by the shape of the platelets in a dielectric surrounding (matrix) and allows one to use the CdSe platelets as a new type of directional emitters e.g. for display technology.

\section{Microscopic origin of the bright plane}

In photoluminescence experiments, electron-hole recombination takes place between bandedge near states, as a population excited in the continuum relaxes fast and radiationless to the band-edge. The top of the valence band in bulk ZB semiconductor has degenerate $h h$ and $l h$ subbands $\left(E_{\Gamma}^{h h}=E_{\Gamma}^{l h}\right)$, see Figure $6(\mathrm{a})$. Since the $h h$ effective mass exceeds the $l h$ 's in Z-direction, ${ }^{35}$ the difference in hole confinement energies leads to the top of the valence band being formed almost exclusively by $h$ h states in CdSe nanoplatelets. (see Methods) The wave function of confined carriers is given by $\Psi_{i}=f_{\nu, n}^{i}\left|u_{i}\right\rangle$, where $i=e, h h, l h$, so indicates electron, heavy hole, light hole or split-off hole, respectively. $f_{\nu, n}^{i}$ is the envelope function, defined over the entire NPL, whose point symmetry is $\nu$ and its main quantum number is $n$. $\left|u_{i}\right\rangle$ is the band-edge periodic function (Bloch function) at the $\Gamma$ point, defined over the unit cell (see SI section F for details).

The probability of interband optical transitions taking place in such a system is pro-

portional to the TDM $\left|\mu_{e j}\right|^{2}=\left|\left\langle f_{\nu_{e}, n_{e}}^{e} \mid f_{\nu_{j}, n_{j}}^{j}\right\rangle\left\langle u_{e}|\boldsymbol{\mu}| u_{j}\right\rangle\right|^{2}$, with $j=h h, l h$, so. The envelope integral provides selection rules, as only transitions fulfilling $\delta_{\nu_{e}, \nu_{j}}$ will be allowed. In turn, the unit cell integral (Bloch part) defines the orientation of the absorbed/emitted light and 

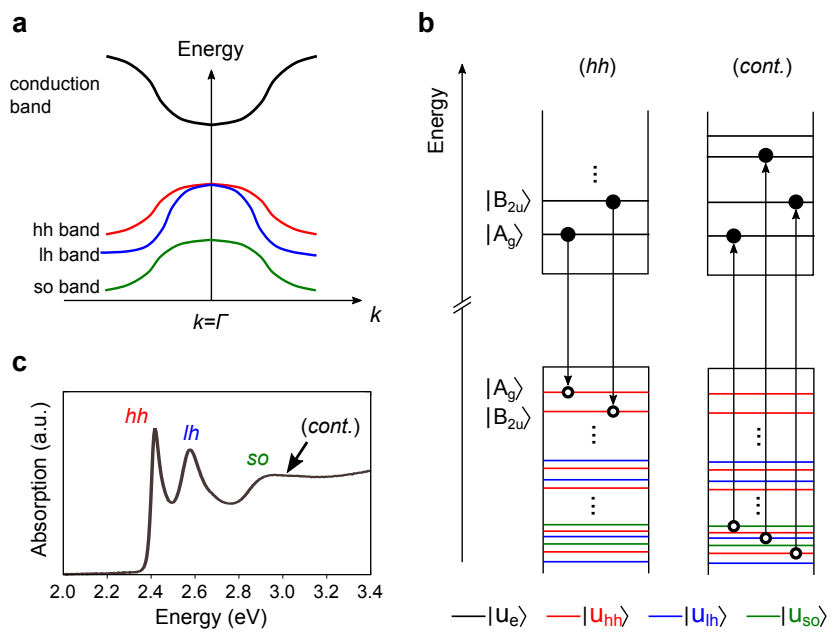

Figure 6: The contribution of different Bloch states determines the isotropic absorption and anisotropic emission of zincblende CdSe nanoplatelets. (a) Bulk zincblende (ZB) semiconductor has degenerate hh and lh subbands. The effective masses along the growth direction are heavier for hh than for lh, so the top of the valence band in NPLs is formed almost exclusively by hh states in CdSe nanoplatelets. (b) Schematic of the conduction band electron and valence band hole energy levels in a rectangular $\left(D_{2 h}\right)$ NPL with ZB crystal structure. Symmetry labels for envelope $|\nu\rangle$ and periodic parts $\left|u_{j}\right\rangle$ of a few states, $|\nu\rangle\left|u_{j}\right\rangle$, are given. The envelope functions, characterized by their basic symmetries $\left(\left|A_{g}\right\rangle\right.$ and $\left.\left|B_{2 u}\right\rangle\right)$, are shown on the left. The Bloch states are given color-coded at the bottom of (b). $(h h)$ : Dipole-allowed near-band-edge transitions (emission). (cont.): Dipole-allowed high energy transitions in absorption. In emission only $h h$ levels (red) are involved, resulting in prevailing out-of-plane emission. In continuum absorption (cont.) $h h, l h$ and split-off holes are equally involved, which results in isotropic absorption. The corresponding transitions of $(h h)$ and (cont.) are indicated in the NPL absorption spectrum in (c). In contrast to the bulk case shown in (a) the confinement lifts the degeneracy of the $h h$ and $l h$.

hence its directionality. Drawing a parallel between the symmetries of a ZB crystal and a hydrogen atom, $\left|u_{e}\right\rangle$ is found to be of $s$-like symmetry, $\left|u_{h h}\right\rangle$ has mixed $p_{x}$ and $p_{y}$ symmetry, and $\left|u_{l h}\right\rangle\left(\left|u_{s o}\right\rangle\right)$ mixed $p_{x}, p_{y}$ and $p_{z}$ character. ${ }^{36}$ Here $x$ and $y$ are in-plane directions, while $z$ is the [001] direction. Therefore, a heavy hole exciton $\Psi=\Psi_{e} \Psi_{h h}$ forms an in-plane electronic dipole, identified as the bright plane.

The integral $\left\langle u_{e}|\boldsymbol{\mu}| u_{h h}\right\rangle$ is non-zero only for $(x, y)$-polarized light, and emission takes place predominantly orthogonal to the NPL surface. By contrast, light hole and splitoff hole excitons have finite dipole projection along $z$, so that $\left\langle u_{e}|\boldsymbol{\mu}| u_{l h}\right\rangle\left(\left\langle u_{e}|\boldsymbol{\mu}| u_{s o}\right\rangle\right)$ is finite in all space directions. (See section G of the SI) This theoretical expectation is in 
excellent agreement with our measured 95\% IP dipole orientation, considering our error margin of $5 \%$. Notice this is independent of the envelope function symmetry, and hence compatible with previous works suggesting emission can have a finite contribution from excited $\left(B_{2 u}\right)$ excitons. ${ }^{13}$ For absorption experiments (Figure 6 (b) column (cont.)), optical transitions include any kind of hole state (or their mixture). On average, this means that the continuum absorption in our CdSe nanoplatelets has an isotropic dipole distribution. (See

also SI section G) This is again in agreement with our experimental results of nearly isotropic transition dipole distribution (70\% in-plane to $30 \%$ out-of-plane) in absorption (ideally $67 \%$ to $33 \%$ ) with an error margin of $\pm 5 \%$.

This adds a new aspect to the well-known exciton funneling in solar cells: In NPLs the contrasting properties of isotropic absorption in the broad continuum, yet anisotropic emission of the heavy hole exciton enable an energy and $k$-space funneling. Energy in the whole continuum is collected isotropically in $\mathrm{k}$-space. It is then concentrated in the lowest states, where it is directionally re-emitted, like an energy and $k$-space concentrator. The presented concept of combining 2D k-space spectroscopy, emission (absorption) directionality and the assessment of the underlying crystal Bloch functions can be readily transferred to other systems such as 2D transition metal dichalcogenides or perovskites to engineer their properties.

\section{Conclusion}

We have demonstrated the directed emission of highly oriented CdSe nanoplatelet monolayers. The heavy hole exciton transition dipoles of the platelets are oriented in a bright plane that coincides with the platelet plane. The resulting out-of-plane directed emission is further favored by local field and optical density of states effects. However, the transition dipole distribution in the platelets' $2 \mathrm{D}$ continuum is isotropic. The observed anisotropic transition dipole distribution for the lowest excitons is directly related to the basic anisotropy of the electronic Bloch states governing the dipole moment. Hence our $k$-space spectroscopy allows 
direct access to the internal Bloch states and opens the possibility to engineer the (directed) emission characteristics by selecting semiconductor crystal systems with appropriate Bloch functions. Hence, we show a route to overcoming basic limitations of the light-extracting efficiency of devices. Here, the electronic anisotropy causing dominant IP emission suppresses losses to non out-coupled (TM) modes (under oblige angles). We can expect a significant increase of the radiative out-coupling efficiency in displays or LED devices. Gain media of oriented platelets in super structures for example are expected to have superior performance over random oriented or spherical nano emitters. Improved inversion conditions and reduction of losses through spontaneous emission might be achieved with high directional gain and flexible pump directions. The combination of isotropic absorption and highly anisotropic emission makes CdSe nanoplatelets an interesting directional emitter for photonic applications like in display technology or lasing, where directed emitters are desirable.

\section{Methods.}

k-space spectroscopy. Oriented monolayers of ZB CdSe nanoplatelets and monolyers of random oriented ZB CdSe dots were obtained by a Langmuir technique on glass substrates (see also Supporting Information). These are introduced in an inverted microscope like setup with a high numeric aperture (1.49) oil immersion objective so that we obtain a three layer system consisting of glass substrate/(nanocrystals and ligands)/air. The sample is excited by the linearly polarized second harmonic of a titanium sapphire laser (Coherent Mira, FWHM $150 \mathrm{fs}, 75.4 \mathrm{MHz}$, excitation density of $0.1 \mathrm{~W} / \mathrm{cm}^{2} \mathrm{CW}$ equivalent) in the continuum of the nanoparticle absorption at $2.99 \mathrm{eV}(415 \mathrm{~nm})$. Angle-dependent confocal excitation is realized by parallel displacement of the beam with respect to the optical axis of the objective. ${ }^{17}$ Following Lieb et al. ${ }^{24}$ and Schuller et al., ${ }^{16}$ the back aperture of the infinity corrected objective is imaged to a sensitive CCD camera through an analyzer (detection polarizer). Details of the samples and preparation can be found in the Supporting Information (SI) section A. A detailed scheme of the setup is shown in figure S3 of the SI. 
The Hertzian radiation pattern of IP or OP dipoles in the focus of the objective can be related to a wave-vector distribution in the back focal plane. Thus the emitted radiation can be decomposed into its Cartesian components $\vec{k}=\overrightarrow{k_{x}}+\overrightarrow{k_{y}}+\overrightarrow{k_{z}}$ leading to a two dimensional projection of the radiation patterns in the $k_{x}-k_{y}$-space. By introducing a polarizer we select electric fields oscillating parallel to the x-z plane. The selection of cuts in the back focal plane (dashed white lines in Fig. 1 a) reduces the analysis to a 2D problem: $k_{x}$ projections contain information about dipoles lying in- and out-of-plane (p-pol projection); $k_{y}$ projections (s-pol) contain only radiation from IP dipoles.

Model analysis of NP emission. We model the emission of the CdSe nanoplatelets at the lowest $2.42 \mathrm{eV}$ heavy hole exciton transition of CdSe platelets and the absorption (into the $2 \mathrm{D}$ continuum at $2.99 \mathrm{eV}$ ). As the oriented CdSe platelets are surrounded by a ligand shell, consisting of oleic acid ligands, our nanoplatelet monolayer samples form an anisotropic effective medium on top of our fused silica substrate. The anisotropic effective medium is modeled by approximating the platelets as oblate ellipsoids, comparable to what is successfully done in Ref. 32 . In the present case we model them as aligned oblate ellipsoids ${ }^{37}$ to account for their in-plane orientation, see section C in SI.

On the glass side (objective) of our heterogeneous system a TDM couples into a different optical density of states as compared to air. For the coupling of IP and OP transition dipoles in the nanocrystal monolayers we use an effective density of states. According to Fermi's Golden Rule, the radiative rate of an emitter is proportional to the product of the Einstein coefficient $A$ (proportional to the TDM $\left|\mu^{2}\right|$ ) and the density of (photon) states $\rho$ yielding $\Gamma_{r}(\omega)=A(\omega) \tilde{\rho}(\omega)=\rho(\omega) \frac{\pi \omega}{3 \hbar} \frac{|\mu(\omega)|^{2}}{\epsilon}$ with $\epsilon=\epsilon_{r} \epsilon_{0} \cdot{ }^{16,24,34,38}$ Using the density of states in a homogeneous medium $\rho_{0}(\omega)=\omega^{2}(\epsilon \mu)^{3 / 2} / \pi$ we decompose the total radiative decay rate and the TDM into IP and OP components. ${ }^{16}$ With the IP and OP Einstein coefficients:

$$
\begin{aligned}
& A_{I P}(\omega)=\rho_{0}(\omega) \frac{\pi \omega}{3 \hbar} N_{r, I P} \frac{\left|\mu_{I P}(\omega)\right|^{2}}{\epsilon} \\
& A_{O P}(\omega)=\rho_{0}(\omega) \frac{\pi \omega}{3 \hbar} N_{r, O P} \frac{\left|\mu_{O P}(\omega)\right|^{2}}{\epsilon}
\end{aligned}
$$


we can relate the PL emission originating from spontaneous heavy hole exciton recombination to the density of photon states in the environment of the emitter. $N_{r, I P}\left|\mu_{I P}(\omega)\right|^{2}$ and $N_{r, O P}\left|\mu_{O P}(\omega)\right|^{2}$ are the IP and OP projections of the dipole strengths with respect to the principle axes of the dipole ellipsoid. $N_{r, I P}$ and $N_{r, O P}$ are the relative weights, which depend on its eccentricity. $\tilde{\rho}^{p, s}\left(\omega, k_{x, y}\right)=\frac{\rho^{p, s}\left(\omega, k_{x, y}\right)}{\rho_{0}(\omega)}$ are the normalized density of states for $\mathrm{p}$ and s polarization. They account for the alteration of the radiative rate in our heterogeneous system with respect to free space.

As the radiative rates are related to the TDMs via eq. 1 we can model the measured angularly dependent emission and absorption characteristics. This allows direct access to the internal distribution of TDMs in IP and OP directions. For a detailed discussion of Fresnel terms and photon density of states the reader is referred to section D of the SI.

Continuum transition dipole moments. For absorption we find the dipole moments of ZB CdSe dot ensemble to be $69 \%$ IP and $31 \%$ OP oriented. This reflects the fully isotropic nature of the absorption transition dipoles of random oriented quantum dot ensembles (e.g. PbSe quantum dots ${ }^{39}$ ). With $70 \%$ IP and $30 \%$ OP orientation the absorption dipole distribution for platelets is likewise isotropic within our experimental error of $5 \%$. The used continuum excitation involves all three bands: heavy- and light- hole as well as the split off band. As shown in the SI section $\mathrm{F}$ the Bloch functions associated to these bands sum up to unity, thus the TDM distribution is isotropic in the continuum.

The observed small deviations of the excitation characteristics of dots and platelets (blue frames in $4 \mathrm{a}$ and $4 \mathrm{c}$ ) are the result of dielectric effects. As mentioned above our nanoplatelet monolayer samples form an anisotropic effective medium ${ }^{32,37}$ differing from the QD monolayer. Further, the QD and NPL monolayers have different thicknesses-determined by the nanocrystal thickness and the length of the oleic acid molecules.

Microscopic origin of the bright plane. In order to allow a full control over the radiative properties of the nanoplatelets, we now discuss the microscopic origin of our observations. The optical anisotropy of ZB NPLs can be explained from their electronic structure. 
One can describe the NPL as a cuboid with dimensions $L_{x} \geq L_{y} \gg L_{z}$. For a qualitative study, we can restrict the analysis to an effective mass description, disregarding band coupling.

Considering the strong confinement in z-direction [001] (perpendicular to the platelet plane) for nanoplatelets, the energy of a carrier $j$ is roughly given by $E^{j} \approx E_{\Gamma}^{j}+\frac{\hbar^{2} \pi^{2}}{2 m_{[001]}^{j} L_{z}^{2}}$. Here $E^{j}$ is the energy splitting from the top of the respective band at the $\Gamma$ point, and $m_{[001]}^{j}$ the effective mass in z-direction. Unlike in wurtzite semiconductors, the top of the valence band in bulk ZB semiconductor has degenerate $h h$ and $l h$ subbands $\left(E_{\Gamma}^{h h}=E_{\Gamma}^{l h}\right)$, see Figure 6 (a). As the names infer the $h h$ effective mass $\left(m_{[001]}^{h h}=0.33\right)$ exceeds the $l h$ 's $\left(m_{[001]}^{l h}=0.13\right)$ in z-direction ${ }^{35}$ so that the top of the valence band is formed almost exclusively by $h h$ states. $l h$ states and split-off holes are split by hundreds of meV, as noted in different experiments ${ }^{3,30}$ and indicated in Fig. 6 (c). Owing to the large energetic separation between $h h$ and $l h$ states, low-energy levels are expected to have weak $h h-l h$ coupling. A diagram of the electron and hole energy levels resulting from the above considerations is plotted in Figure 6 (b). The levels are labeled by the corresponding periodic function as well as the envelope function symmetry related to the $D_{2 h}$ point group of the rectangular nanoplatelet.

Electron-hole recombination in photoluminescence experiments takes place between states near the band edges. As shown in Figure. 6 (b) column $(h h)$, this involves only emission from the heavy hole to which all excited higher excitons and e-h pairs cool down. According to the results presented in the main text, the emission dipoles of these $h h$ excitons lie in the $\mathrm{x}-\mathrm{y}$ plane and are thus IP. This prediction is in excellent agreement with our measured $95 \%$ IP dipole orientation, considering our error margin of $5 \%$.

Radiation patterns. The distance from the origin to a point on the surface of the 3D objects in Figure 5(b) and (c) is proportional to the irradiance transmitted through the surface of a unit sphere at a given solid angle $I(\theta, \phi)$. The monolayer ensemble of NPLs (QDs) is placed in the center of the unit sphere and aligned to the $\mathrm{x}-\mathrm{y}$ plane. To compare the radiation characteristics of the NPL and QD monolayer ensembles quantitatively we 
normalize to their total emitted power $I_{t o t}=\iint I(\theta, \phi) \sin \theta \mathrm{d} \theta \mathrm{d} \phi$. For a detailed discussion on local field factors and radiation patterns see SI section E.

\section{Acknowledgements}

R.S., U.W. and A.W.A acknowledge DFG grants WO477-1/32 and AC290-1/1 and 2/1. J.I.C. acknowledges support from MINECO project CTQ2014-60178-P and UJI project P11B2014-24, M.A. from the CHEMREAGENTS program, A.A. from BRFFI grant No. X16M020.

\section{Author contributions}

J.H., R.S., A.V.P. and N.G. performed the measurements, A.V.P., A.A., A.M. and M.A. made the samples, J.H., R.S., N.G. and A.W.A. analysed, modeled and interpreted the data, J.I.C. contributed theoretical interpretation, J.H., R.S., N.G, J.I.C., N.O. and A.W.A wrote the manuscript. U.W. contributed to the discussion.

\section{Additional information}

Supplementary information is available in the online version of the paper. Reprints and permission information is available online at www.nature.com/reprints. Correspondence and requests for materials should be addressed to A.W.A.

\section{Data availability statement}

All relevant data (shown in Figures 3, 4, S1, S2 and S5) are available from the authors (A.W.A.). 


\section{References}

(1) Basu, B. K. Theory of Optical Processes in Semiconductors; Oxford Univ. Press, Oxford, 1997.

(2) Ithurria, S.; Dubertret, B. Quasi 2D Colloidal CdSe Platelets with Thicknesses Controlled at the Atomic Level. J. Am. Chem. Soc. 2008, 130, 16504-16505.

(3) Achtstein, A. W. et al. Electronic structure and exciton-phonon interaction in twodimensional colloidal CdSe nanosheets. Nano Lett. 2012, 12, 3151-7.

(4) Joo, J.; Son, J. S.; Kwon, S. G.; Yu, J. H.; Hyeon, T. Low-Temperature Solution-Phase Synthesis of Quantum Well Structured CdSe Nanoribbons. J. Am. Chem. Soc. 2006, $128,5632-5633$.

(5) Wang, Q.; Kalantar-Zadeh, K.; Kis, A.; Coleman, J.; Strano, M. Electronics and optoelectronics of two-dimensional transition metal dichalcogenides. Nat. Mat. 2012,

(6) Kaasbjerg, K.; Thygesen, K. S.; Jacobsen, K. W. Phonon-limited mobility in $n$-type single-layer $\mathrm{MoS}_{2}$ from first principles. Phys. Rev. B 2012, 85, 115317.

(7) Perera, M. M. et al. Improved Carrier Mobility in Few-Layer MoS2 Field-Effect Transistors with Ionic-Liquid Gating. ACS Nano 2013, 7, 4449-4458.

(8) Benchamekh, R. et al. Tight-binding calculations of image-charge effects in colloidal nanoscale platelets of CdSe. Phys. Rev. B 2014, 89, 035307.

(9) Cheiwchanchamnangij, T.; Lambrecht, W. R. L. Quasiparticle band structure calculation of monolayer, bilayer, and bulk $\mathrm{MoS}_{2}$. Phys. Rev. B 2012, 85, 205302.

(10) Scott, R. et al. Time-Resolved Stark Spectroscopy in CdSe Nanoplatelets: Exciton Binding Energy, Polarizability, and Field-Dependent Radiative Rates. Nano Lett. 2016, $16,6576-6583$. 
(11) Chernikov, A. et al. Exciton Binding Energy and Nonhydrogenic Rydberg Series in Monolayer $W S_{2}$. Phys. Rev. Lett. 2014, 113, 076802.

(12) Naeem, A. et al. Giant exciton oscillator strength and radiatively limited dephasing in two-dimensional platelets. Phys. Rev. B 2015, 91, 121302.

(13) Achtstein, A. W. et al. p-State Luminescence in CdSe Nanoplatelets: Role of Lateral Confinement and a Longitudinal Optical Phonon Bottleneck. Phys. Rev. Lett. 2016, $116,116802$.

(14) C. Poellmann, C. et al. Resonant internal quantum transitions and femtosecond radiative decay of excitons in monolayer WSe2. Nat. Mat. 2016, 14, 889.

(15) Wang, H. et al. Radiative lifetimes of excitons and trions in monolayers of the metal dichalcogenide $\mathrm{MoS}_{2}$. Phys. Rev. B 2016, 93, 045407.

(16) Schuller, J. A. et al. Orientation of luminescent excitons in layered nanomaterials. Nat. Nanotechnol. 2013, 8, 271-276.

(17) Brown, S. J.; Schlitz, R. A.; Chabinyc, M. L.; Schuller, J. A. Morphology-dependent optical anisotropies in then-type polymer P(NDI2OD-T2). Physical Review B 2016, 94.

(18) Pukhov, K. K.; Basiev, T. T.; Orlovskii, Y. V. Spontaneous emission in dielectric nanoparticles. JETP Lett. 2008, 88, 12-18.

(19) Chuang, S. L.; Chang, C. S. k·p method for strained wurtzite semiconductors. Phys. Rev. B 1996, 54, 2491.

(20) Empedocles, S.; Neuhauser, R.; Bawendi, M. Three-dimensional orientation measurements of symmetric single chromophores using polarization microscopy. Nature $\mathbf{1 9 9 9}$, 399, 126-130. 
(21) Koberling, F. et al. Fluorescence Anisotropy and Crystal Structure of Individual Semiconductor Nanocrystals. J. Phys. Chem. B 2003, 10 \%, 7463.

(22) Rodina, A.; Efros, A. Effect of Dielectric Confinement on Optical Properties of Colloidal Nanostructures. JETP Lett. 2016, 149, 641-655.

(23) Novotny, L.; Hecht, B. Principles of Nano-Optics; Cambridge University Press, Cambrigde, 2006.

(24) Lieb, M. A.; Zavislan, J. M.; Novotny, L. Single-molecule orientations determined by direct emission pattern imaging. J. Opt. Soc. Am. B 2004, 21, 1210.

(25) Mooney, J.; Kambhampati, P. Get the Basics Right: Jacobian Conversion of Wavelength and Energy Scales for Quantitative Analysis of Emission Spectra. J. Phys. Chem. Lett. 2013, 4, 3316-3318.

(26) Kim, D. Y. et al. Overcoming the fundamental light-extraction efficiency limitations of deep ultraviolet light-emitting diodes by utilizing transverse-magnetic-dominant emission. Light: Science \& Applications 2015, 4, e263.

(27) Brütting, W.; Frischeisen, J.; Schmidt, T. D.; Scholz, B. J.; Mayr, C. Device efficiency of organic light-emitting diodes: Progress by improved light outcoupling. physica status solidi (a) 2013, 210, 44-65.

(28) Schmidt, T. D. et al. Evidence for non-isotropic emitter orientation in a red phosphorescent organic light-emitting diode and its implications for determining the emitter ̌s radiative quantum efficiency. Applied Physics Letters 2011, 99, 163302.

(29) Chen, X. et al. Angular distribution of polarized light and its effect on light extraction efficiency in AlGaN deep-ultraviolet light-emitting diodes. Optics Express 2016, 24, A935. 
(30) Ithurria, S. et al. Colloidal nanoplatelets with two-dimensional electronic structure. Nat. Mater. 2011, 10, 936-941.

(31) The Merck Index- An Encyclopedia of Chemicals, White House Station NY, 2006. https://www.rsc.org/Merck-Index.

(32) Achtstein, A. W. et al. Linear Absorption in CdSe Nanoplates: Thickness and Lateral Size Dependency of the Intrinsic Absorption. J. Phys. Chem. C 2015, 119, 2015620161.

(33) Dolgaleva, K.; Boyd, R. W. Local-field effects in nanostructured photonic materials. Adv. Opt. Photonics 2012, 4, 1.

(34) Taminiau, T. H.; Karaveli, S.; van Hulst, N. F.; Zia, R. Quantifying the magnetic nature of light emission. Nat. Commun. 2012, 3, 979.

(35) Adachi, S. Handbook on physical properties of semiconductors; Kluwer Academic Publishers, Boston, 2004.

(36) Yu, P.; Cardona, M. Fundamentals of Semiconductors; Springer, Berlin, 1996.

(37) Sihvola, A.; Kong, J. Effective permittivity of dielectric mixtures. IEEE Trans. Geosci. Remote Sens 1988, 26, 420-429.

(38) Loudon, R. The Quantum Theory of Light, 3rd ed.; Oxford Univ. Press, Oxford, 2000.

(39) Cunningham, P. D.; Boercker, J. E.; Placencia, D.; Tischler, J. G. Anisotropic Absorption in PbSe Nanorods. ACS Nano 2014, 8, 581-590. 